\title{
Osmotic stress and water isotope effects in kinesin-1 gliding motility assays
}

Steve Koch, U. New Mexico Dept. Physics and Center for High Technology Materials (CHTM)
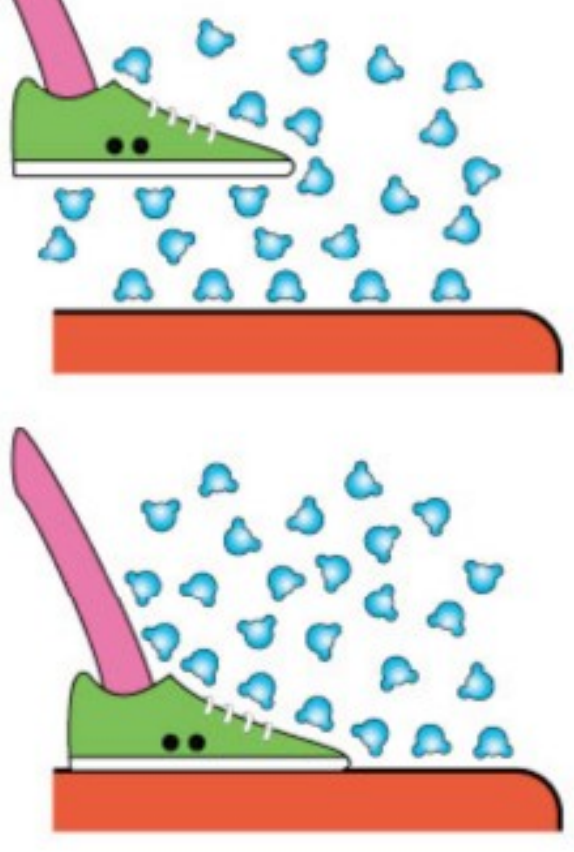

Thermodynamics and kinetics of molecular motors workshop Santa Fe, May 20, 2010

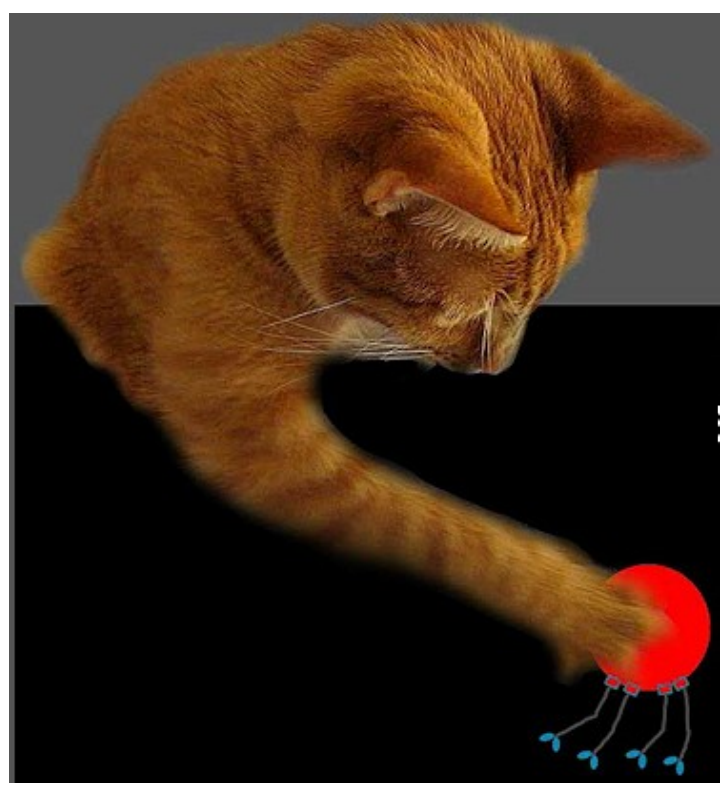




\section{Acknowledgments}

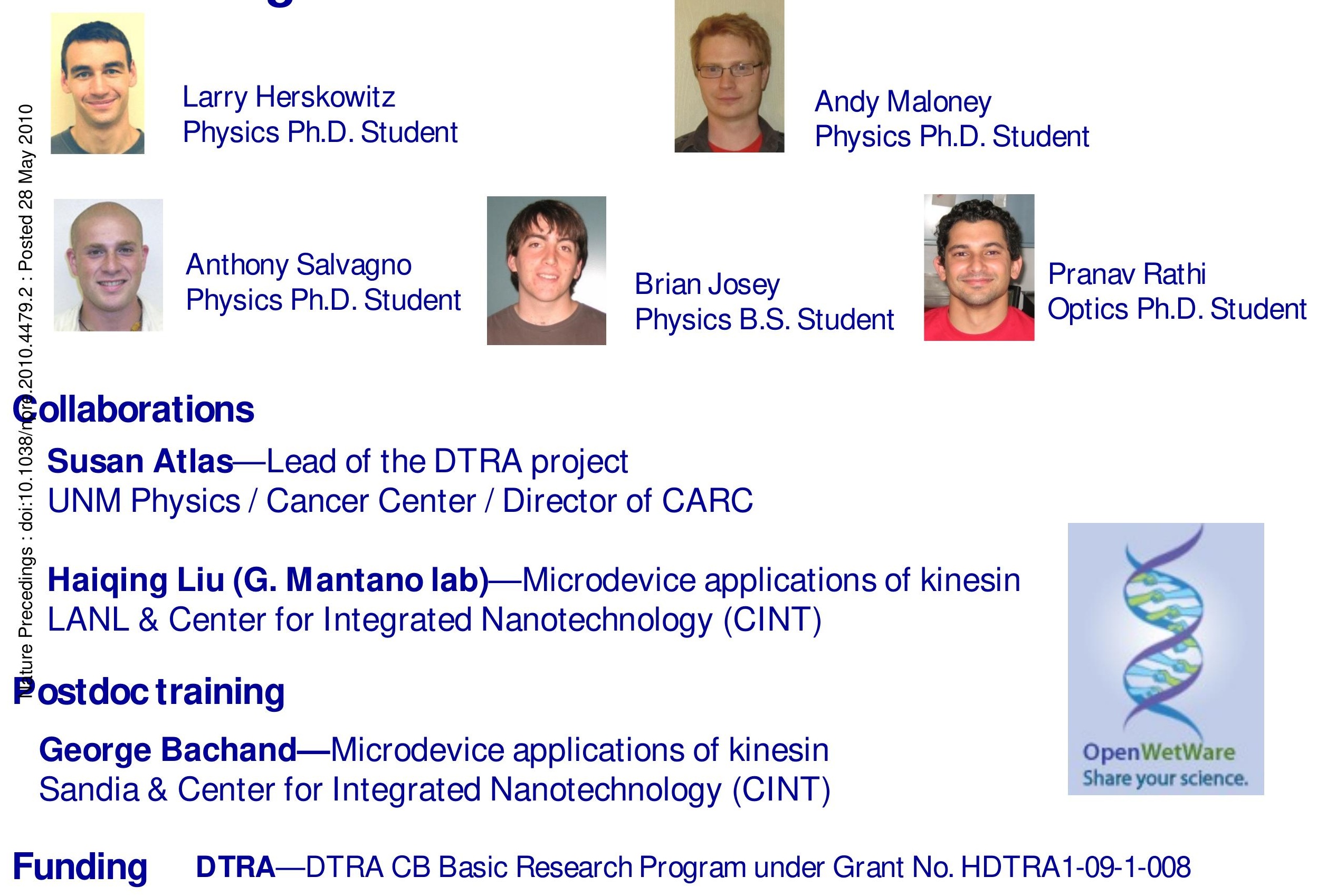


1. Overview of our gliding assay approach

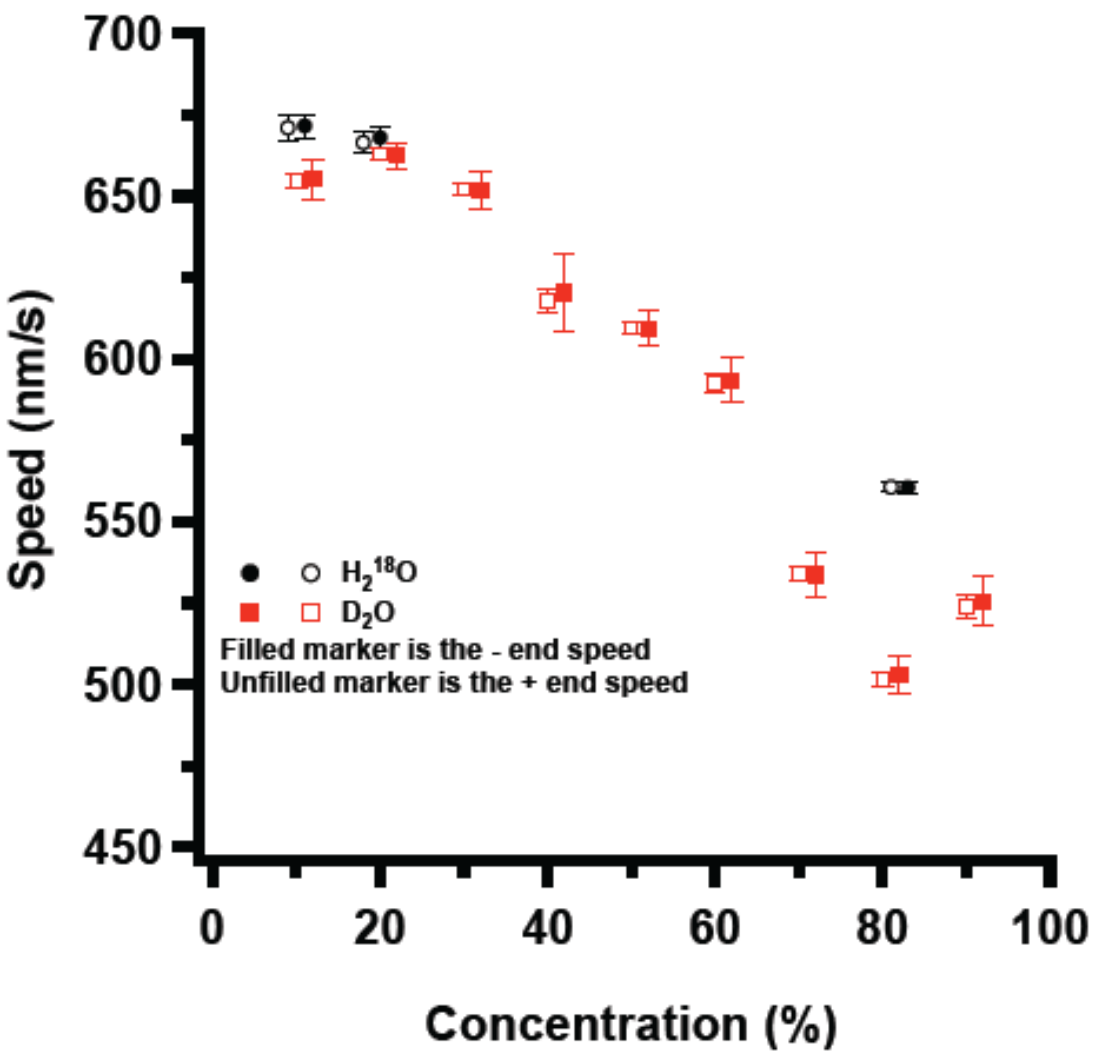

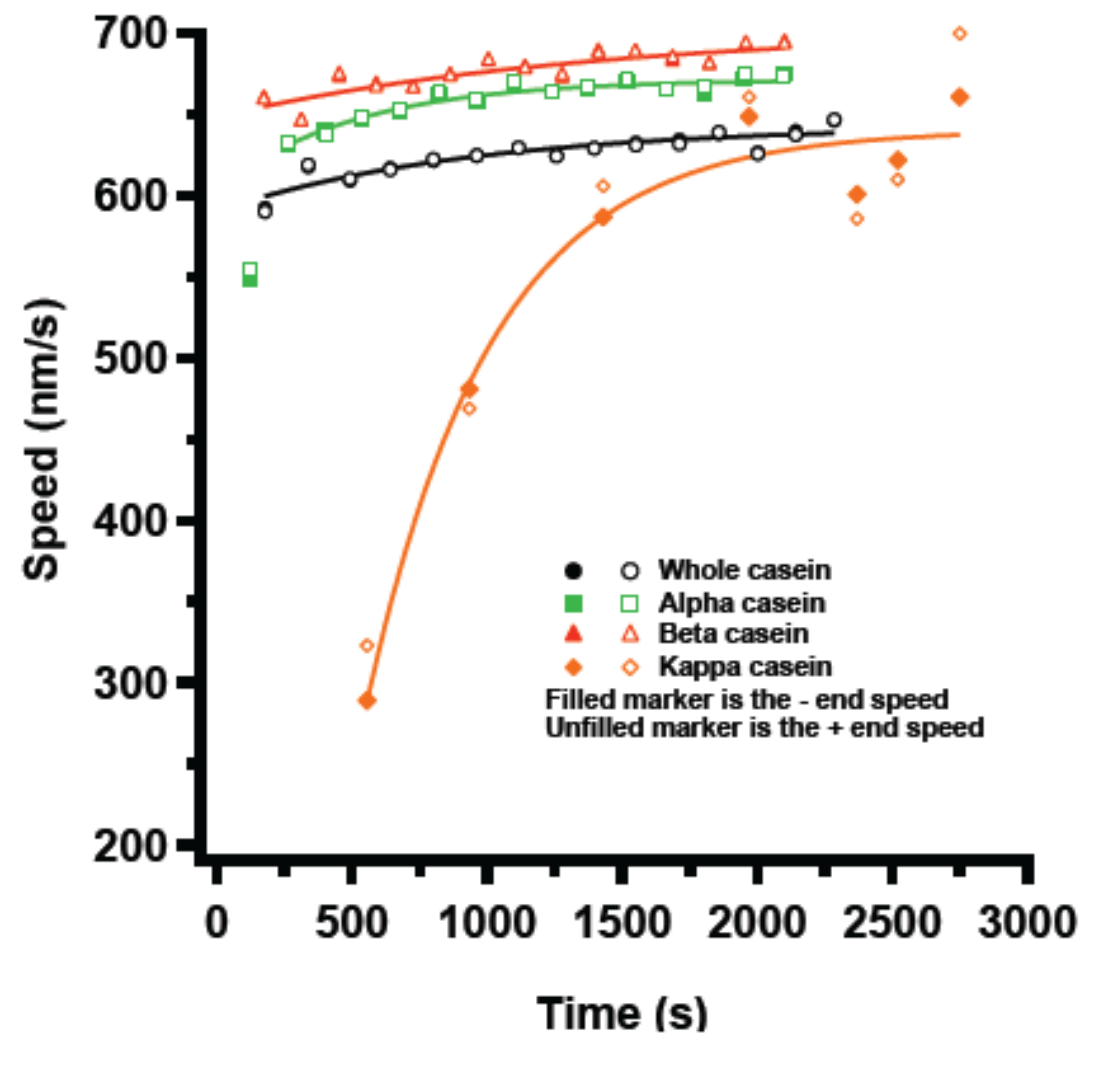

2. Preliminary results isotope and osmotic stress effects in kinesin-1 gliding assays. 
Kinesin / MT introduction

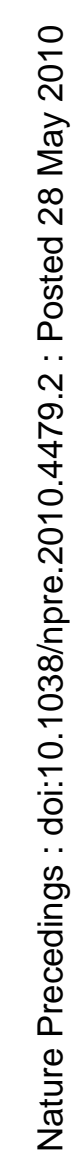

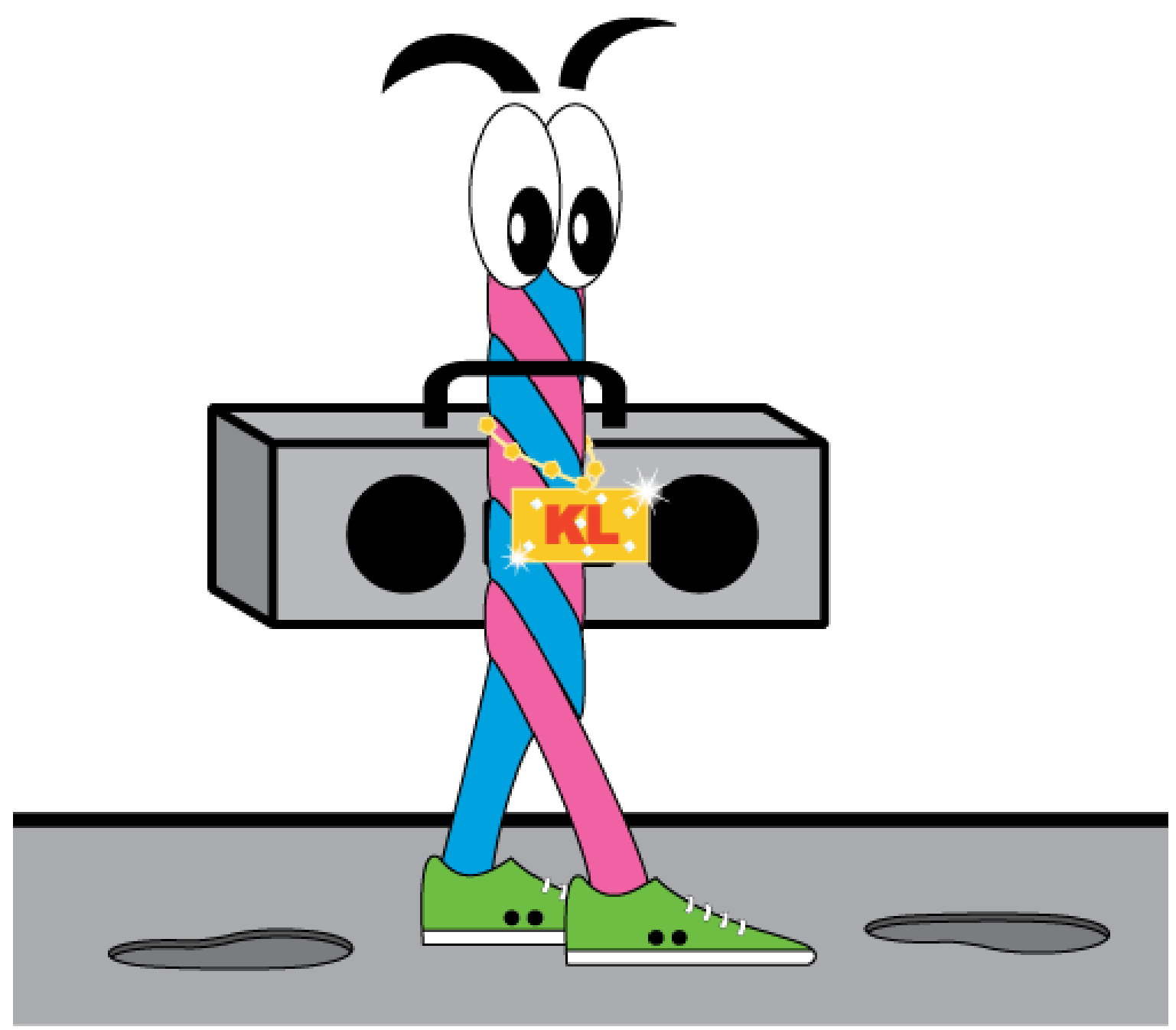

'Kiney' 


\section{Gliding motility assay}
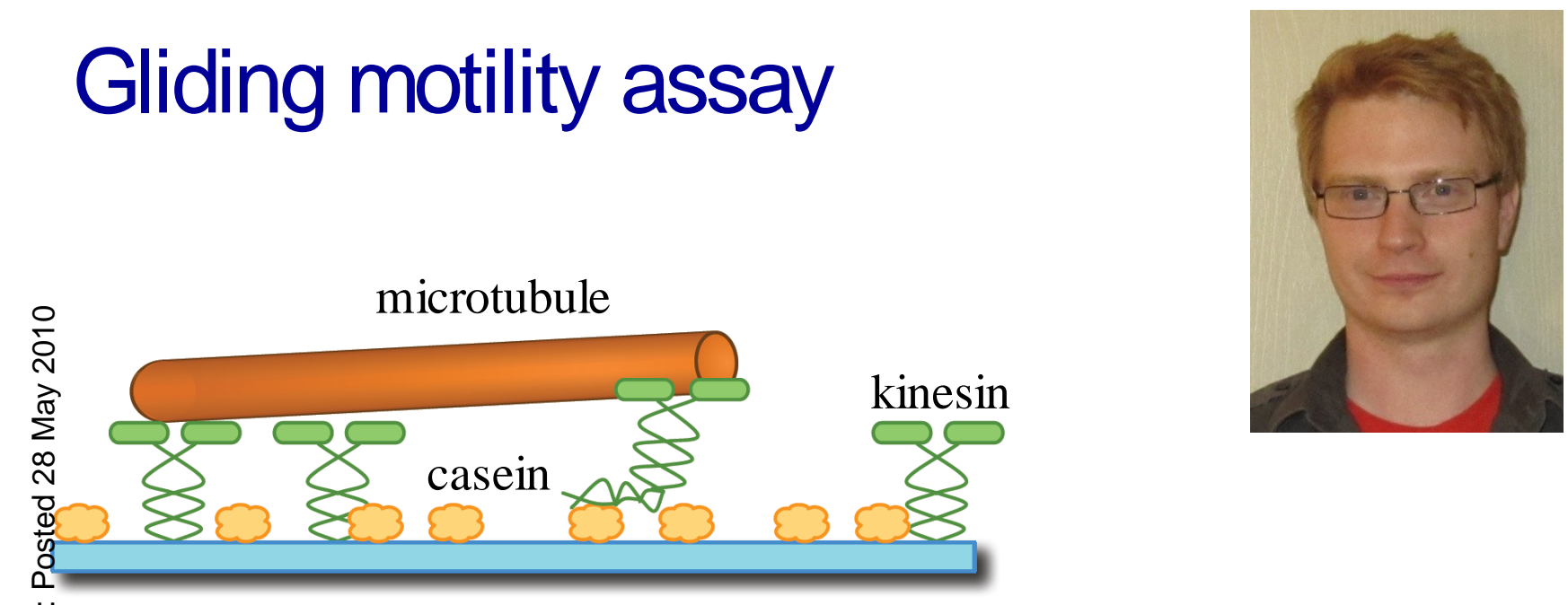

\section{Andy Maloney}

Gliding motility assays Caseins Heavy Water Osmotic Stress

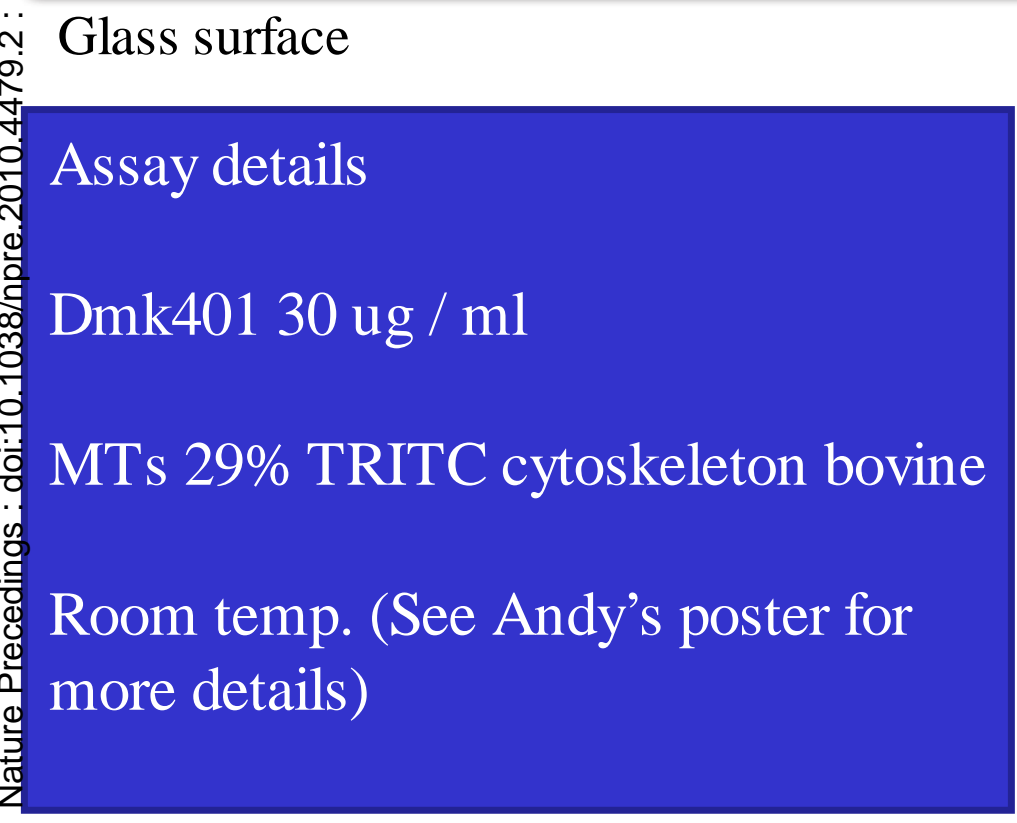

Video: Beta casein 6x speed; false-colored

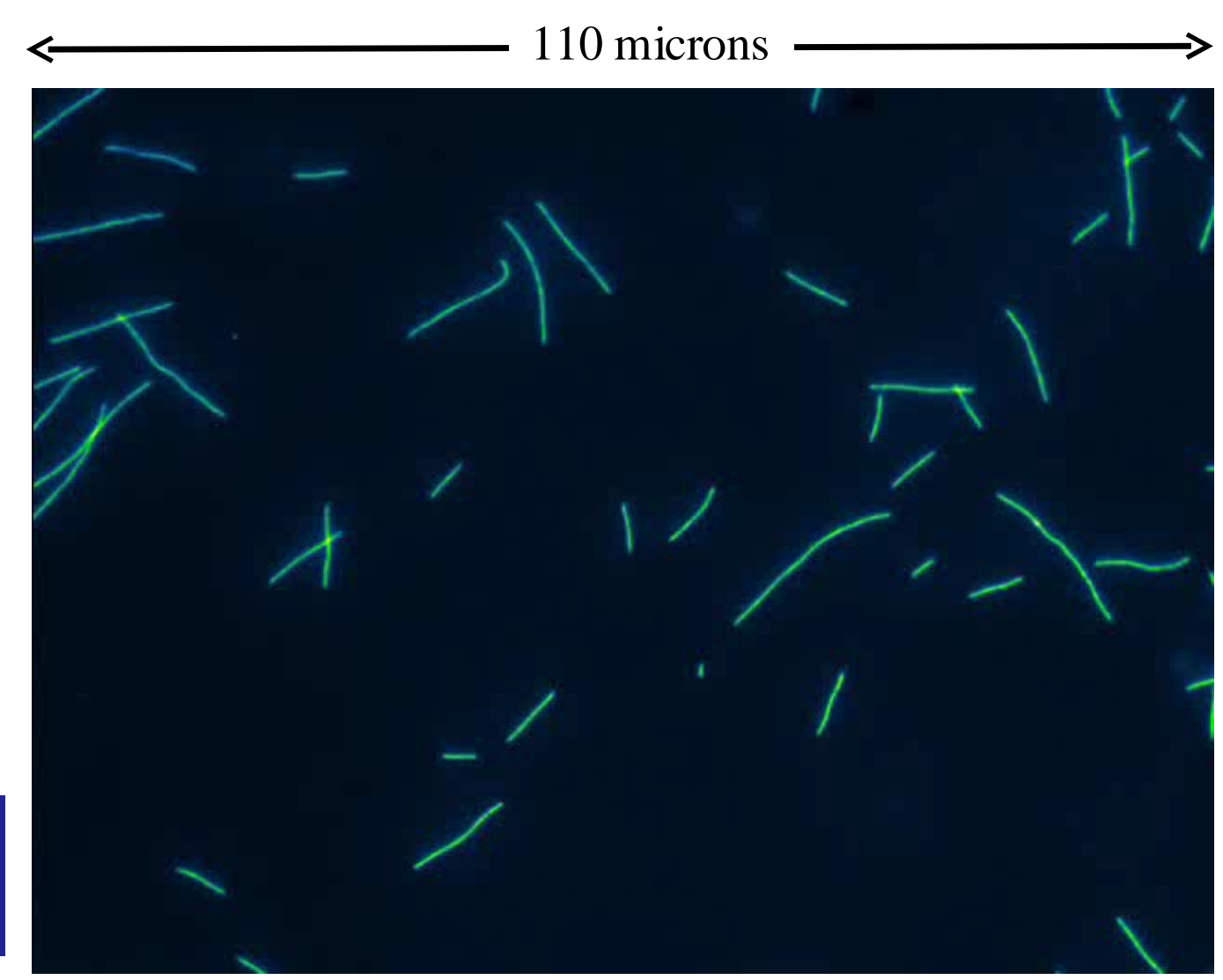




\section{Gliding motility assay}

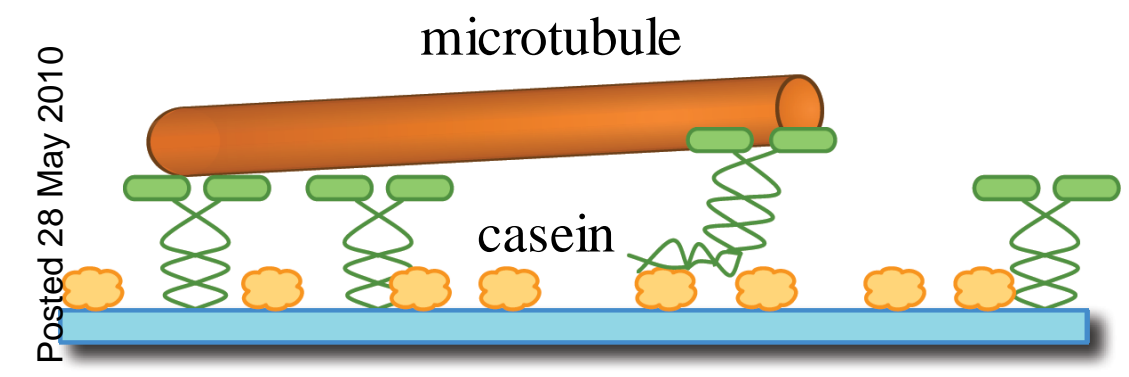

\section{Glass surface}

$\stackrel{8}{2}$

$\stackrel{8}{8}$

के

Andy can finish one sample in

oु 1 hour from start to finish

글

30 minutes of data, 9,000 images

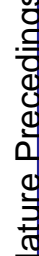

Primary measurement: speed

寻

Also other qualitative measurements

Advantage: lots of data quickly; robust assay

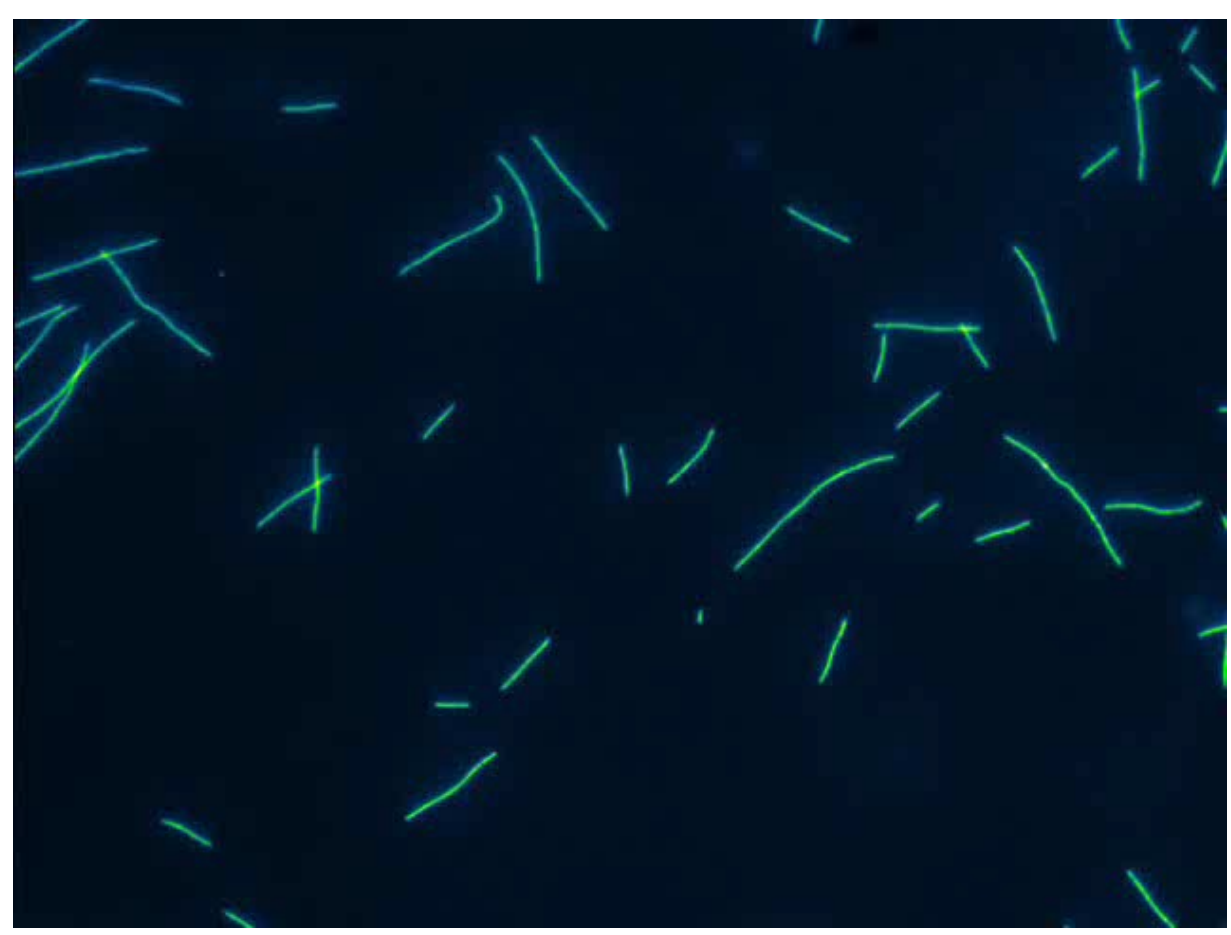




\section{We've developed an open-source, automated microtubule tracking and speed analysis platform}

1. Automated Tracking of MTs

Ts identified with NI Vision 7.1

Template for

pattern matching

Larry Herskowitz Image tracking Stochastic Simulation Image Simulation

\&

Segmenting Routines

osition and Angle of MT ends

found via image

pattern matching

,Y position versus time recorded

for all MTs in folder heirarchy

30 minutes of raw data processed

in approx.

1 hour using 4 cores

See Larry's poster today for details

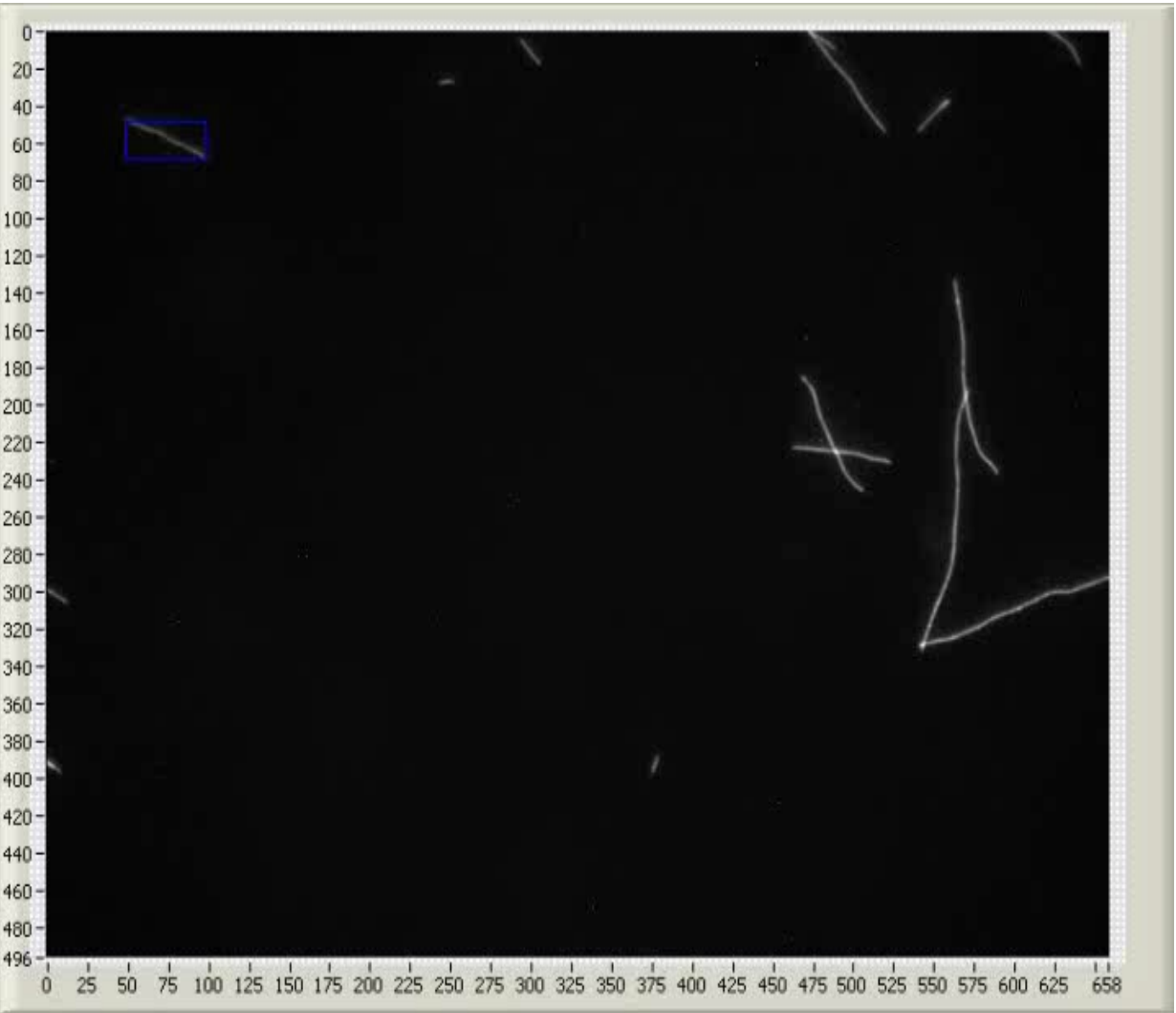




\section{Automated speed analysis}

\section{Kernel density estimation of speed probability distribution function (PDF)}

Gaviasian kernel, bandwidth 50 nm / s
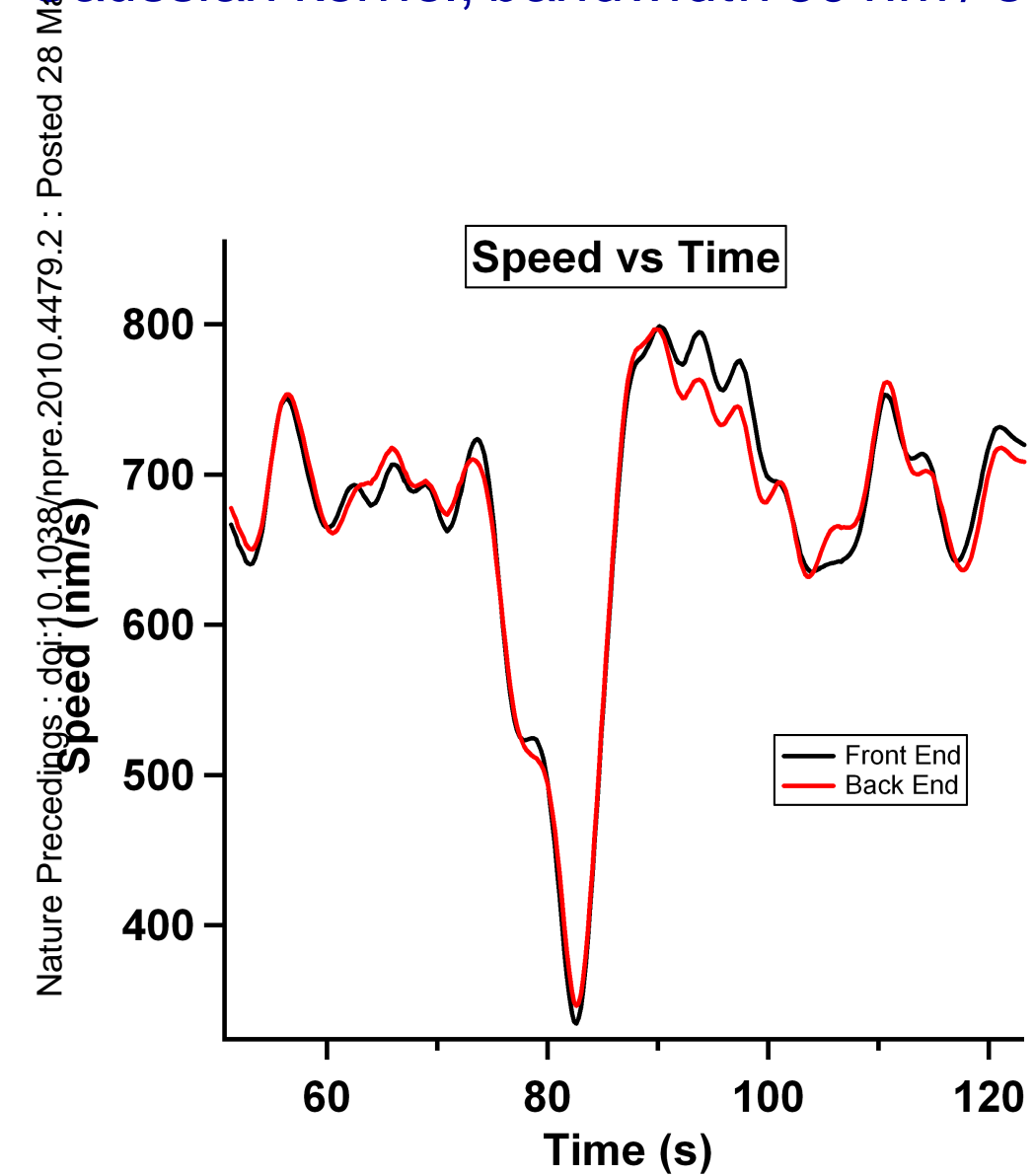

Silverman 1986 Density Estimations
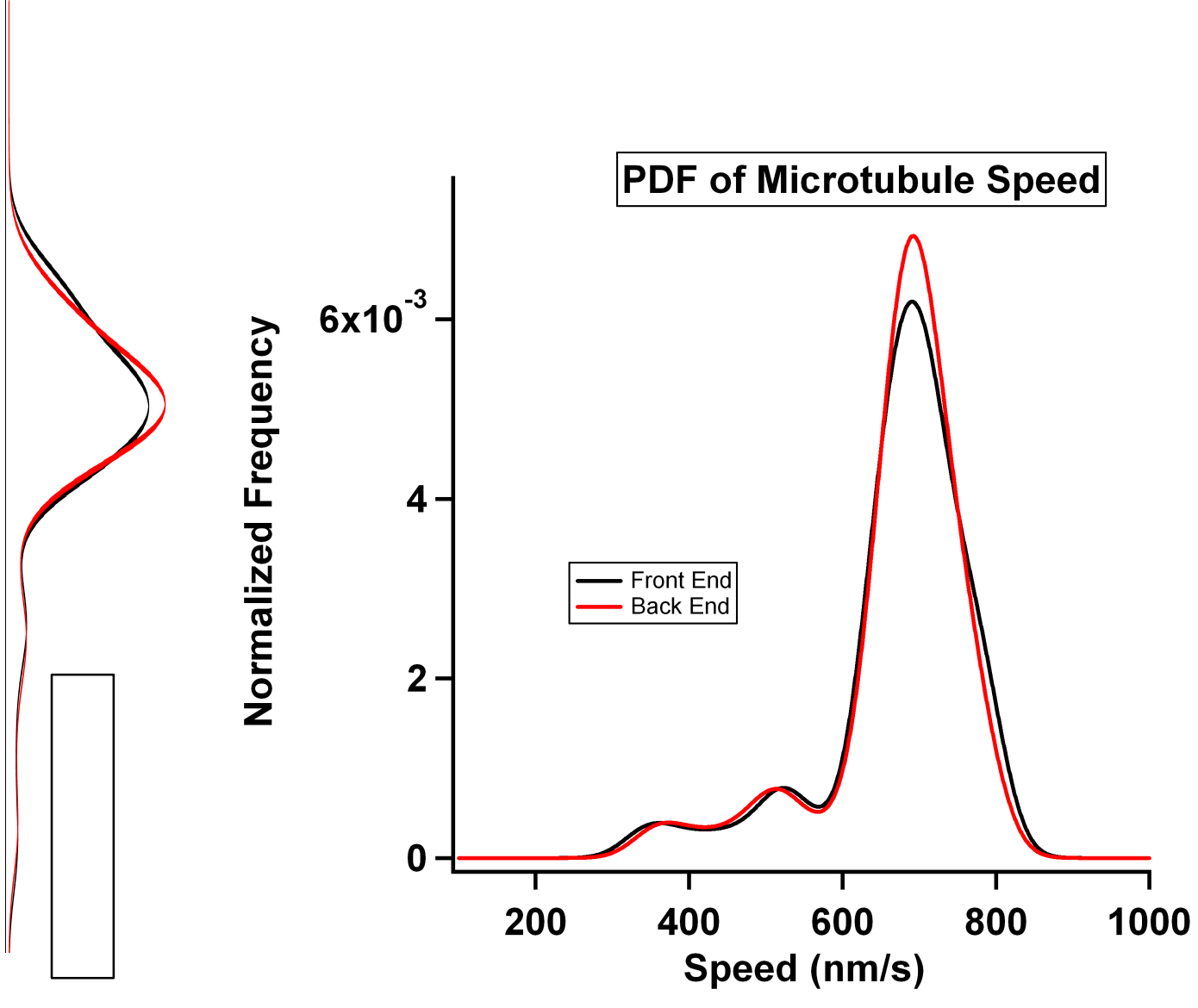


\section{Automated speed analysis}

C. Kernel density estimation of speed probability distribution function (PDF)

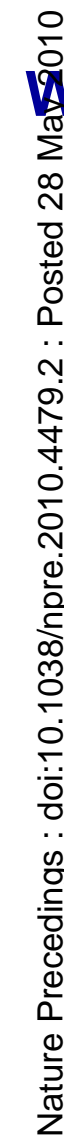

e checked our algorithm using simulated microtubule image series* constant gliding speed $\mathrm{w} /$ pause of random duration
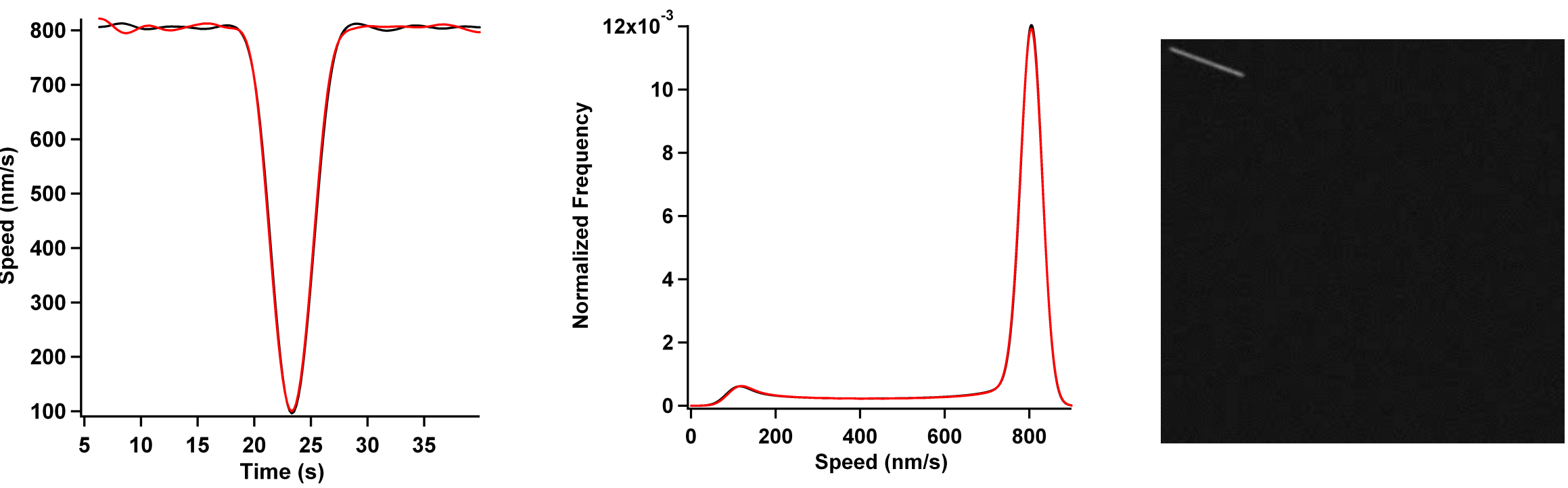

* Herskowitz and Koch (2010) Nature Precedings http://dx.doi.org/10.1038/npre.2010.4374.1 


\section{Automated speed analysis}

C. Kernel density estimation of speed probability distribution function (PDF)

/e checked our algorithm using simulated microtubule image series constant gliding speed $\mathrm{w} /$ pause of random duration
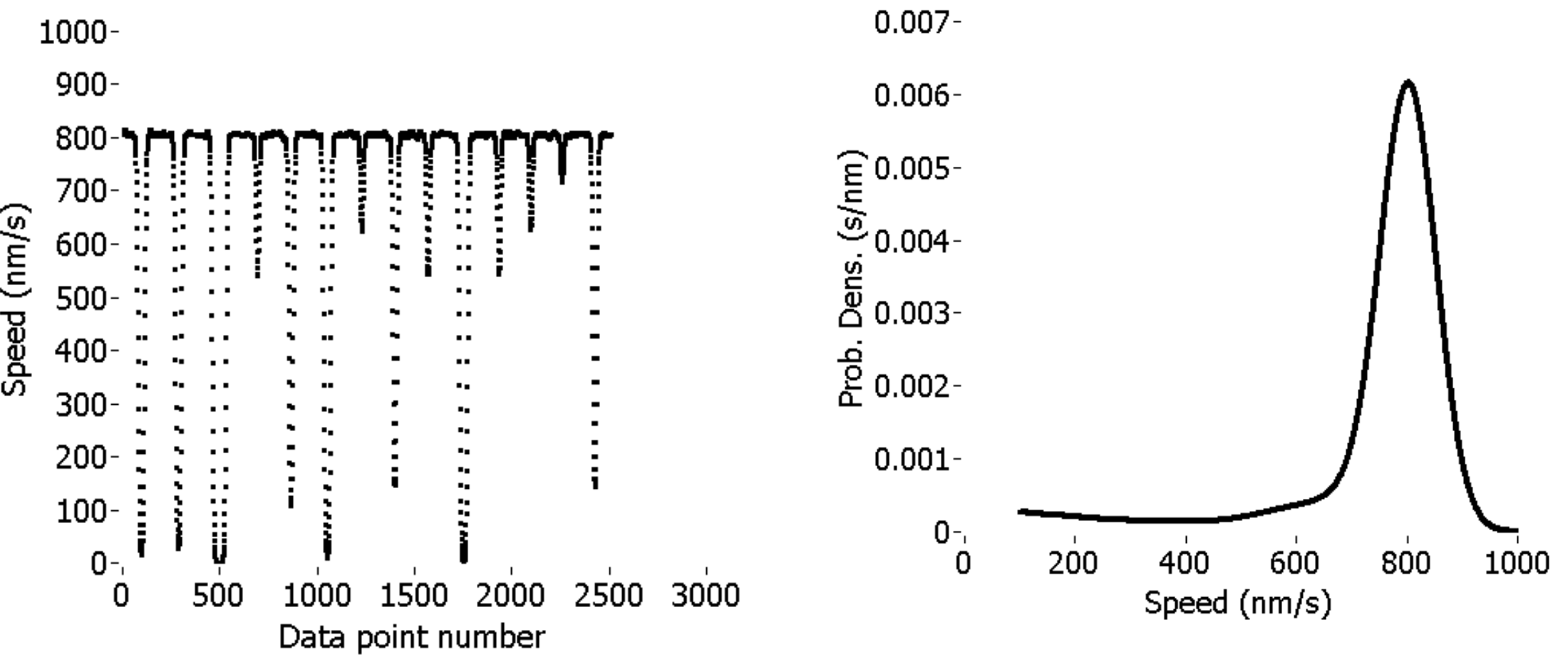

Calculated speed $805.4+/-0.2 \mathrm{~nm} / \mathrm{s} \quad(\mathrm{N}=15)$

Actual gliding speed $807 \mathrm{~nm} / \mathrm{s}$

Pauses probably bias data a bit ... more significant with wider bandwidth 


\section{Automated speed analysis}

C. Speed PDF analysis not greatly affected by pauses or tracking errors

Permits automated speed determination for pooled MT tracks for given condition
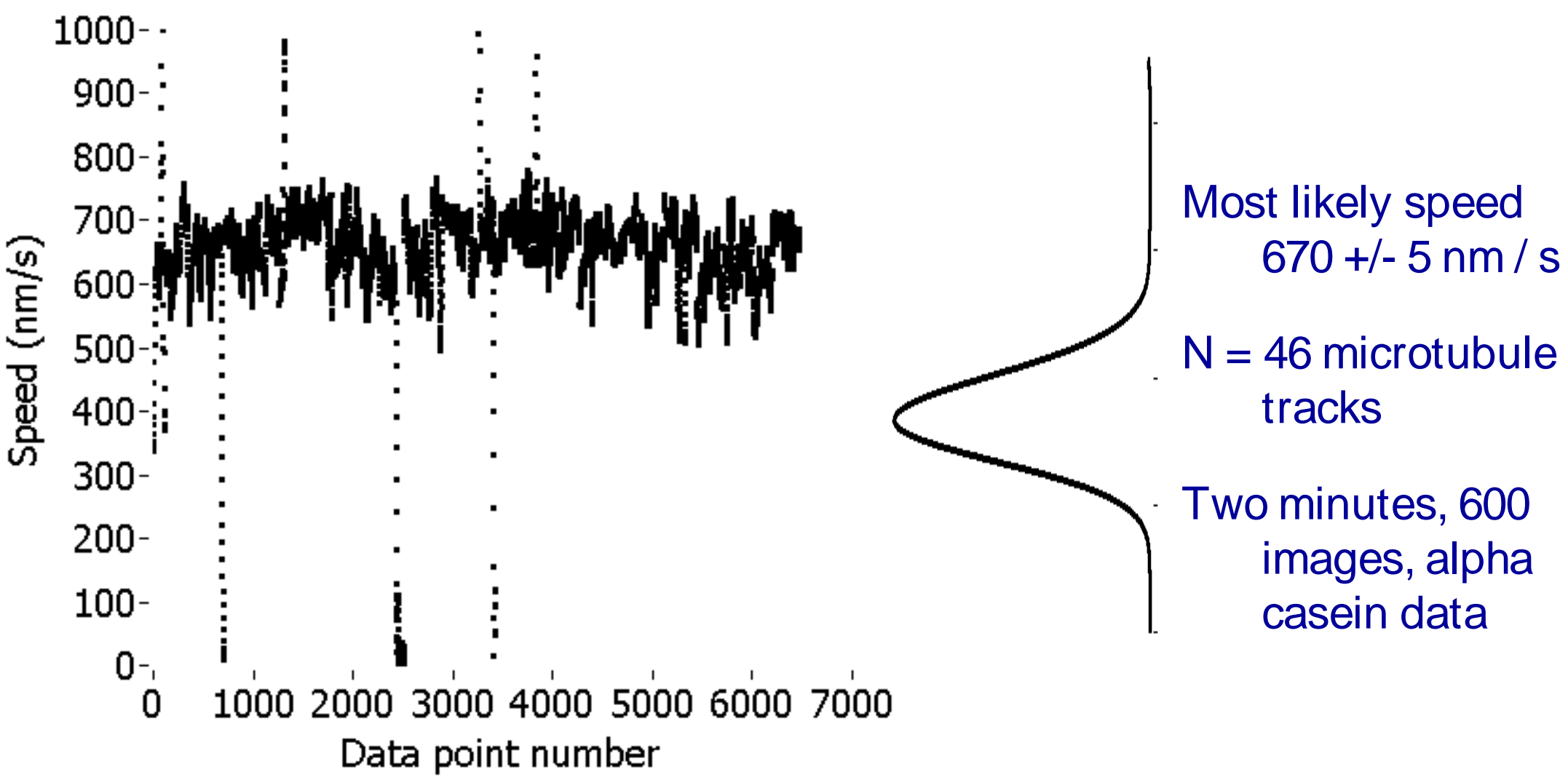

Data point number 


\section{Automated speed analysis}

C. Speed PDF analysis not greatly affected by pauses or tracking errors

Permits automated speed determination for pooled MT tracks for given condition

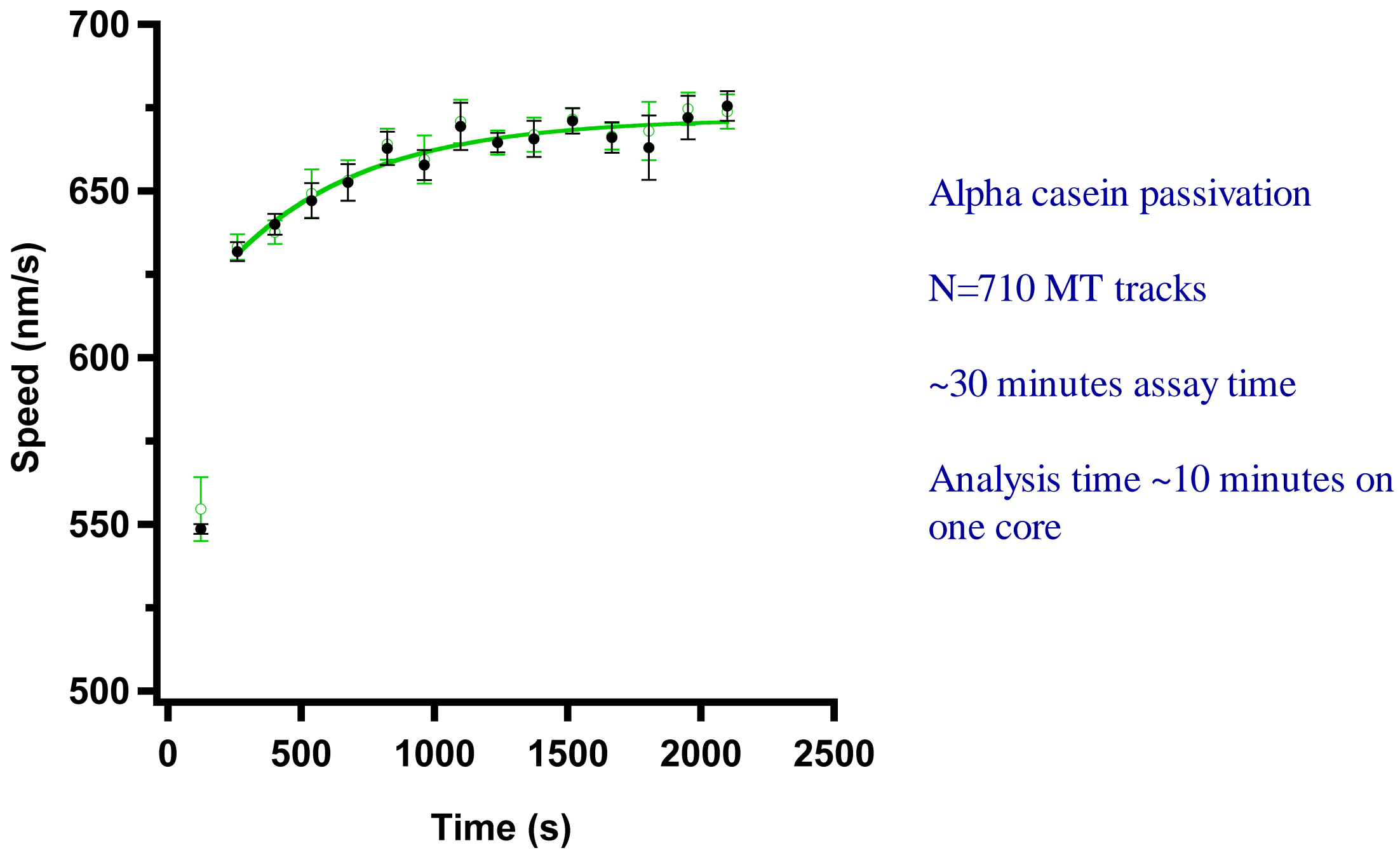




\section{Automated speed analysis}

Speed gradually increases with time for all casein varieties

- Intrinsic speed differences between varieties

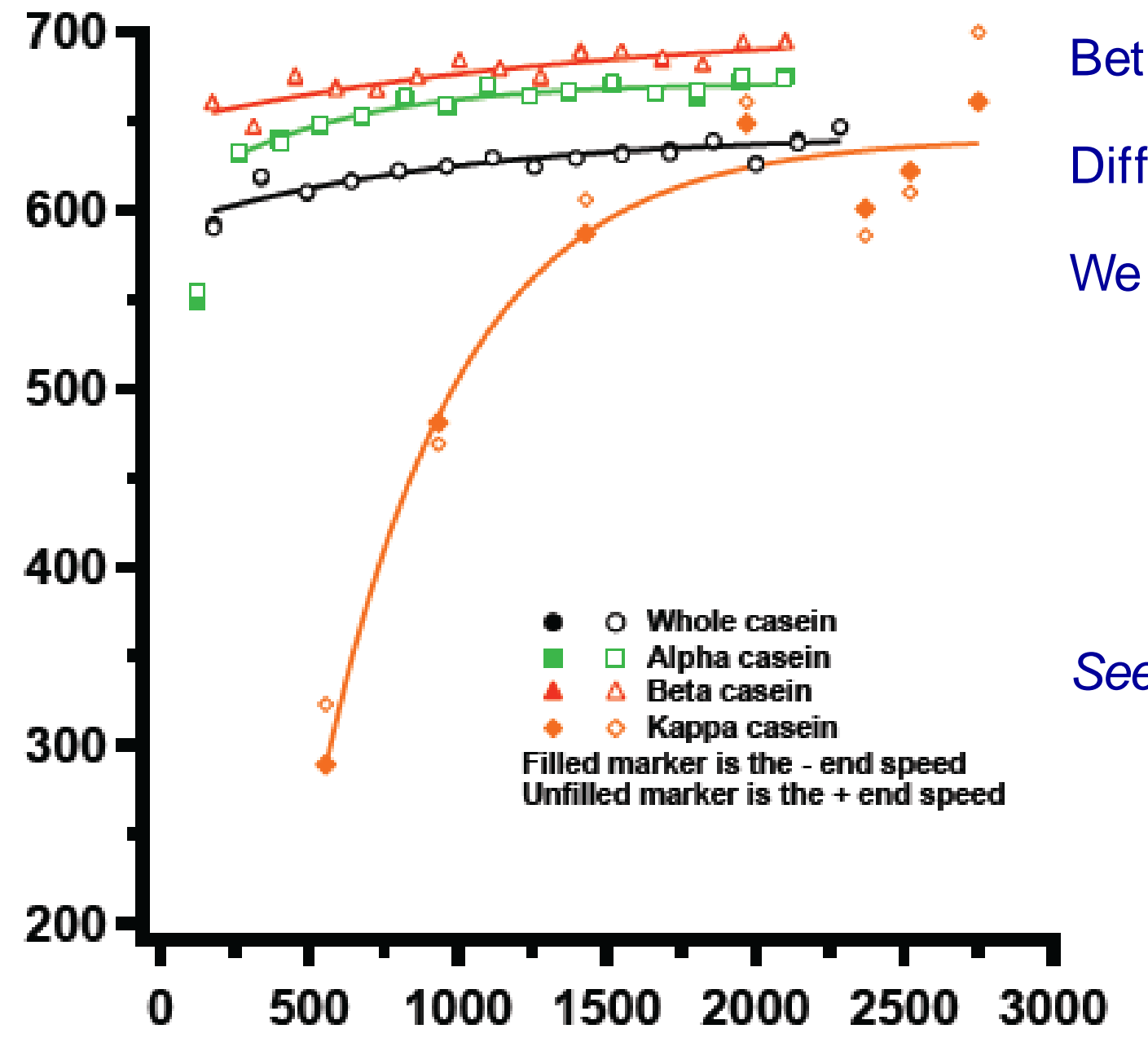

Beta casein highest speed

Differences in active motor density?

We chose alpha casein and these analysis methods for subsequent isotope and osmotic stress studies

See Andy Maloney's poster for more details

See also: Verma, V., Hancock, W. O., \& Catchmark, J. M. (2008). Journal of biological engineering, 2, 14. doi: 10.1186/1754-1611-2-14.

Time (s) 
Varying the properties of water may be a useful knob for studying kinesin

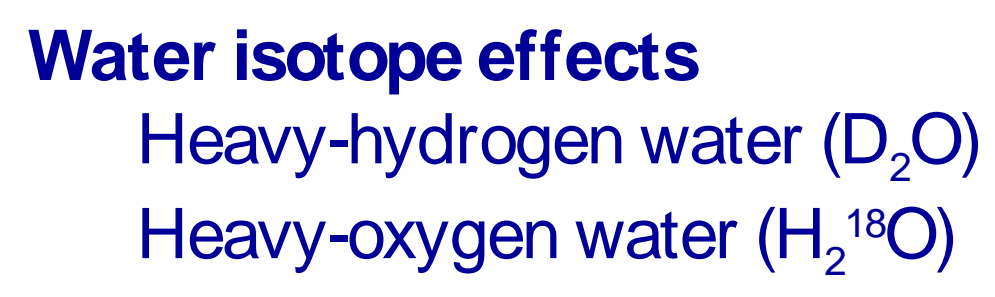

\section{Osmotic stress}

Betaine, Future work: other osmolytes: proline, sucrose, etc.

Future: denaturants

May be a method for probing large-surface area interactions between kinesin and tubulin 
Osmotic stress dramatically increases lifetime of bound molecular complexes

An example from protein-DNA interactions
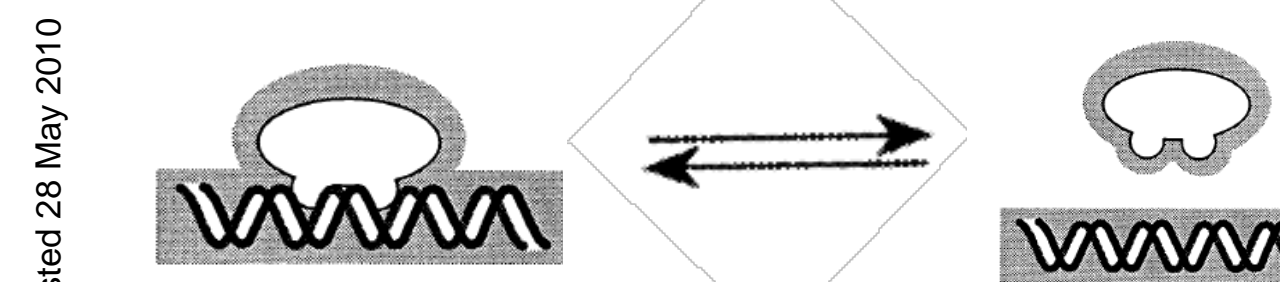

vacocan

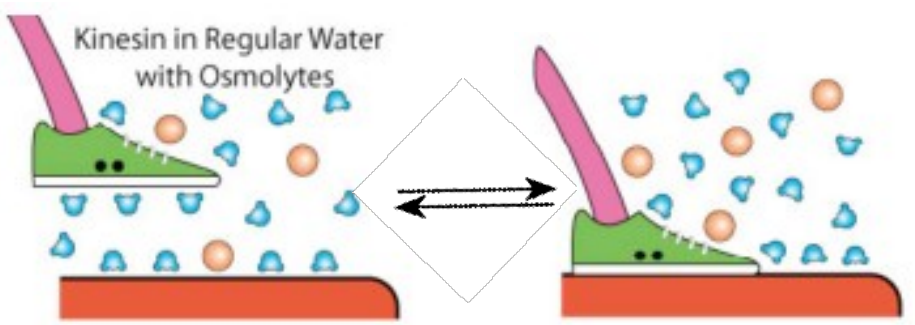

No kinesin studies yet; A few myosin studies

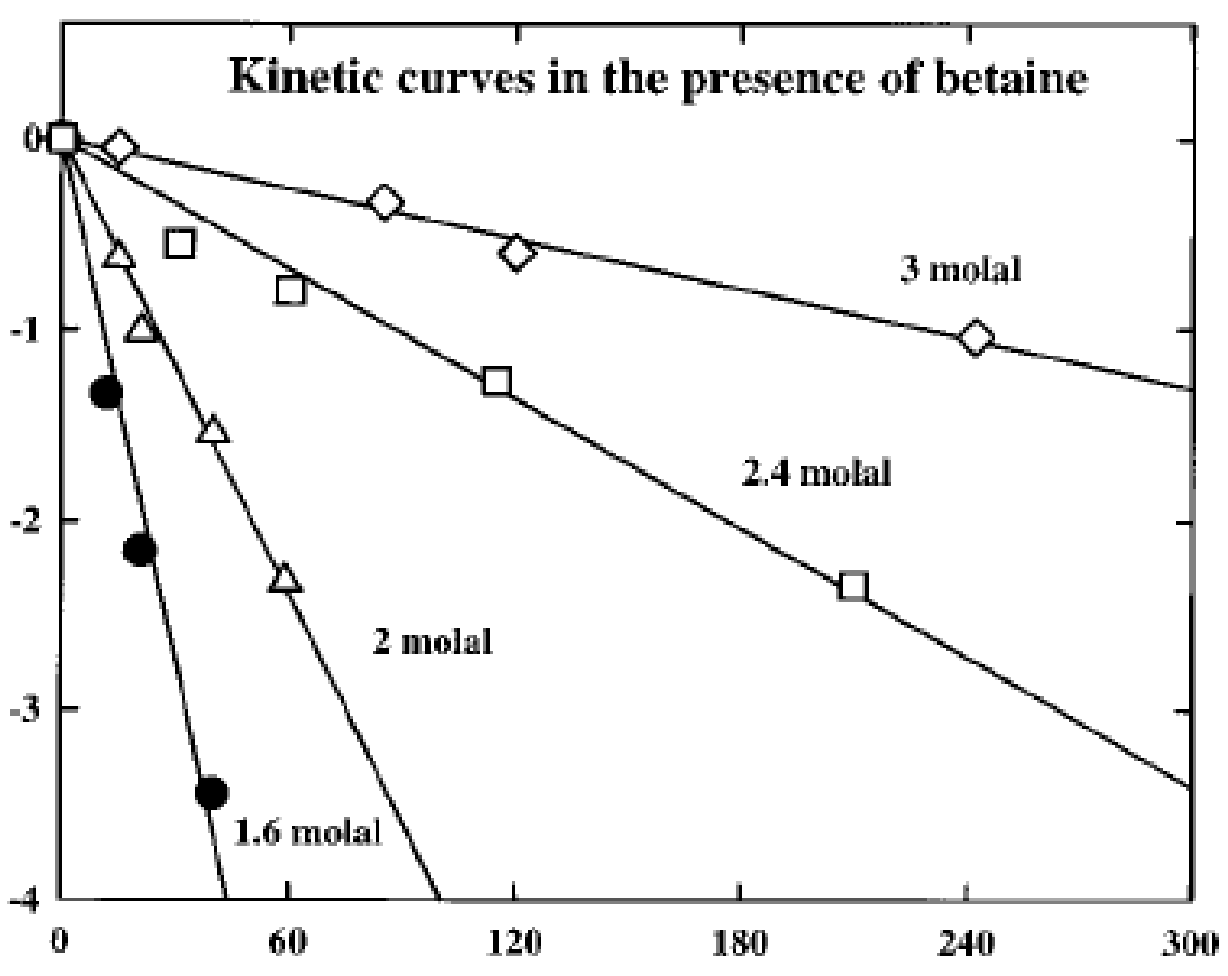

Sidorova and Rau

Biopolymers, Vol. 53, 363-368 (2000)

$\frac{d\left[\ln \left(k_{\text {off }}\right)\right]}{d[\text { osmolal }]}=-\frac{\Delta N_{w}}{55.6}$

Off rate reduced by $>100$ fold

time, min. 


\section{Heavy-hydrogen water (D2O) has been used to probe the actomyosin system}

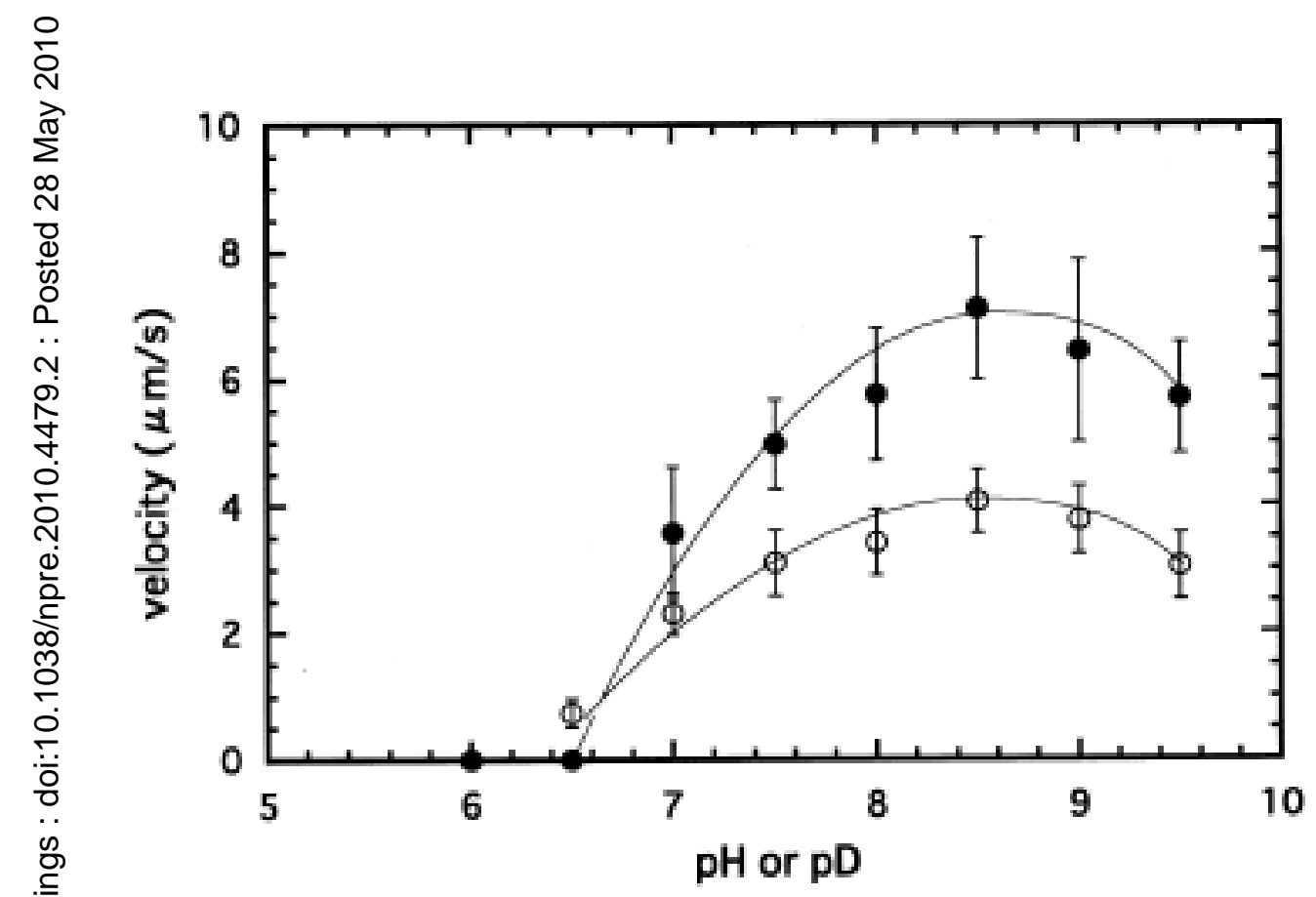

ig. 1. Dependence of the actin sliding velocity on $\mathrm{pH}(\bullet)$ and

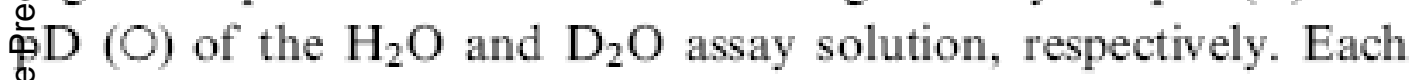
Eata point represents the average velocity of 11 different filaments, and the vertical bar shows the standard deviation. The curves were drawn by eye.
The authors list among many possible explanations, an effect on myosin-actin affinity.

How should water isotopes affect kinesin motility?

Answers from molecular dynamics?

Chaen, S., et al (2001). Effect of deuterium oxide on actomyosin motility in vitro. Biochimica et biophysica acta, 1506(3), 21823. Pub med: 11779555. 


\section{Another reason we're looking at water effects is our partnership with Atlas / Valone labs}

Susan Atlas (UNM; Pl of our DTRA project) and Steve Valone (LANL) are developing the "Charge-Transfer Embedded-Atom Model (CT-EAM)" force field for MD simulations of kinesin and other biomolecules.

Incorporates "correct" quantum mechanics so that charge transfer can be handled.

One of the first benefits of CT-EAM will be better simulation of water dynamics... including for differing isotopes.

Big disconnect between length and time scales for gliding assay and MD

But both can look at water dynamics.

MD: How many hydrating water molecules?

How do water dynamics change with isotope?
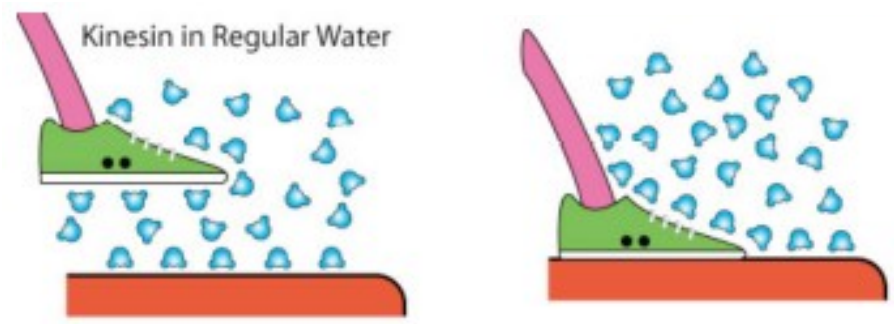


\section{Stochastic simulation can help us understand how water might affect kinesin motility}

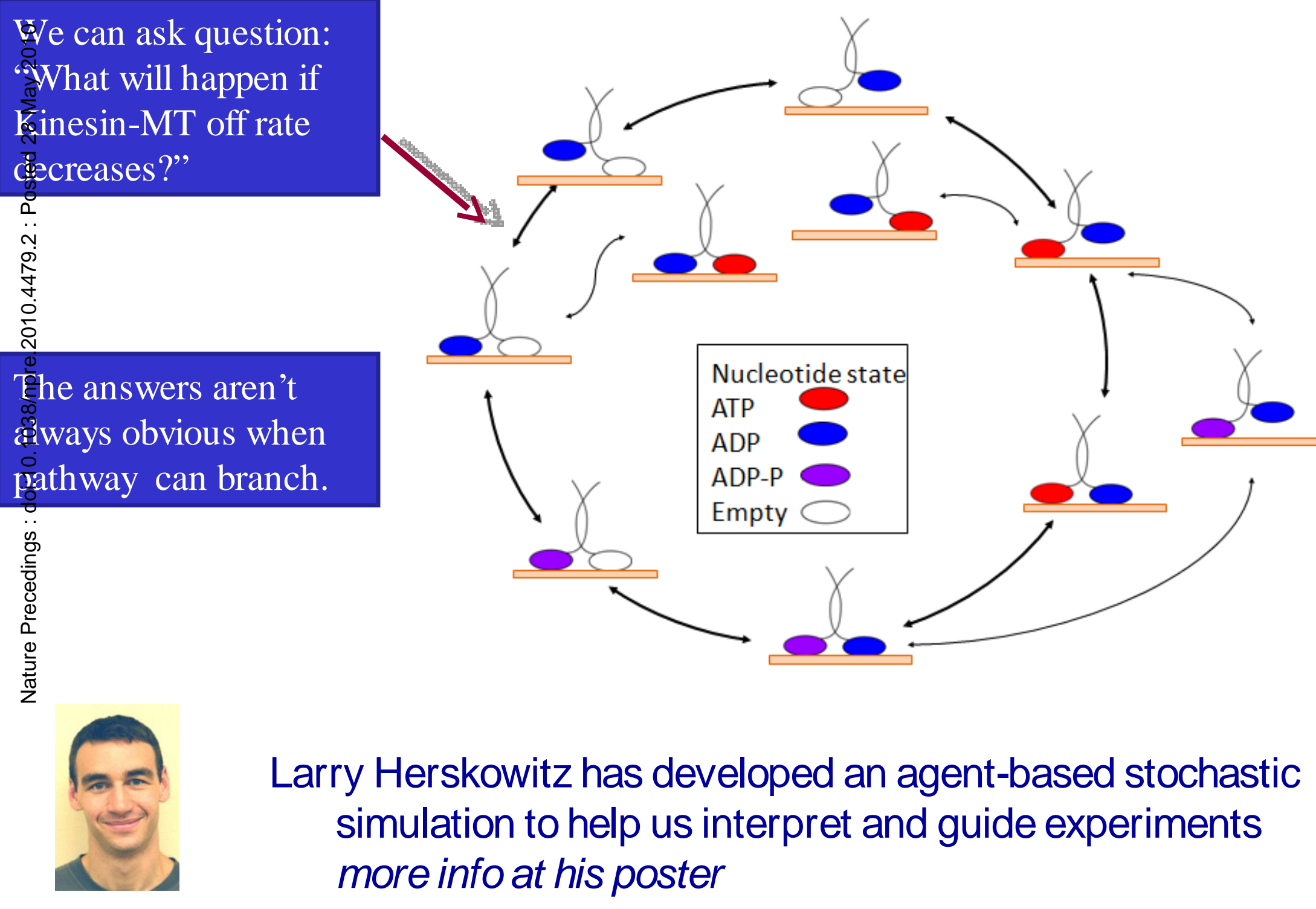




\section{Stochastic simulation can help us understand how water might affect kinesin motility}

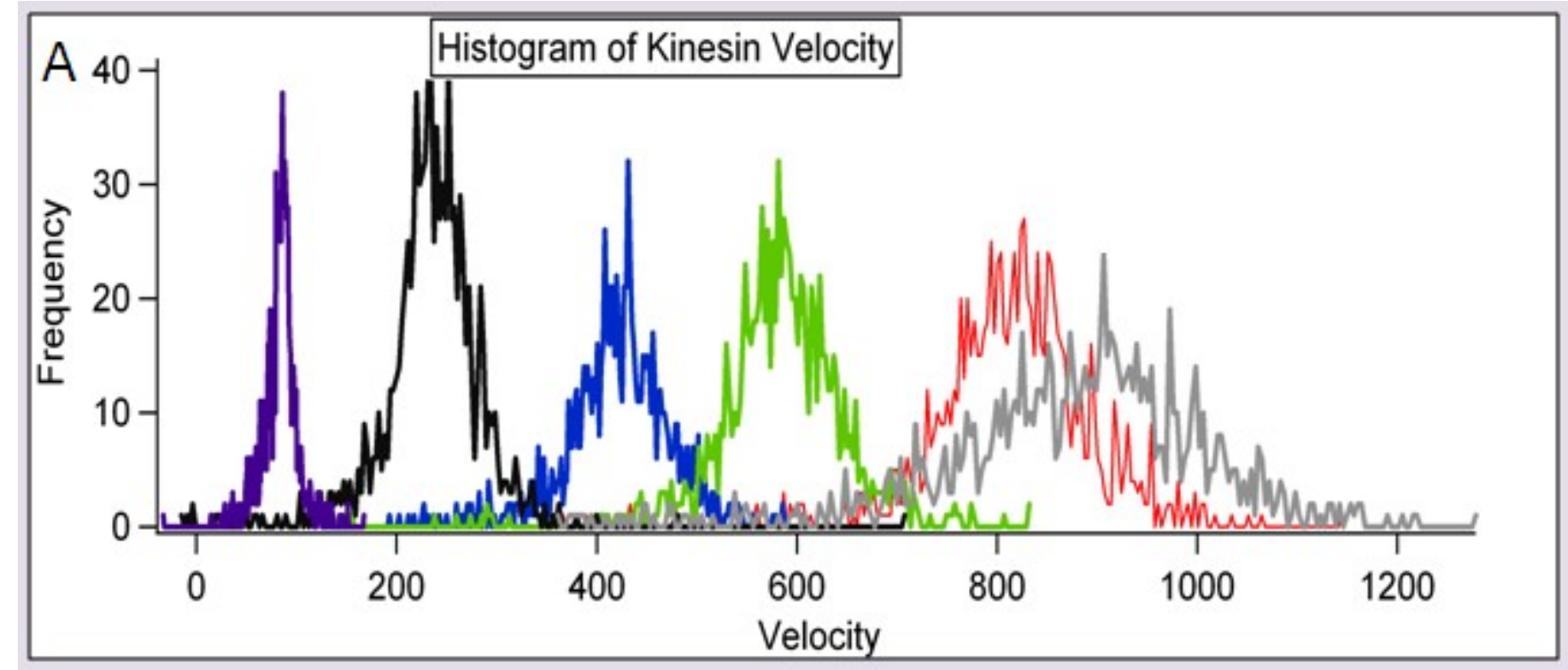

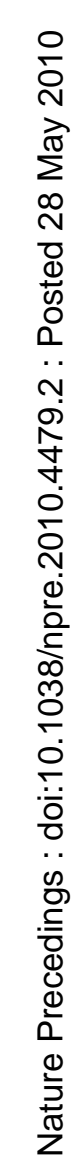

- Default Parameters

2 times Default on rate; $1 / 2$ times Default off rate

- 3 times Default on rate; $1 / 3$ times Default off rate

— 5 times Default on rate; $1 / 5$ times Default off rate

— 10 times Default on rate; $1 / 10$ times Default off rate

- 1/2 times Default on rate; 2 times Default off rate

Stochastic simulations tentatively indicate that increased osmotic pressure or increased water mass would decrease kinesin speed. 


\section{Other features seen in D2O assays}

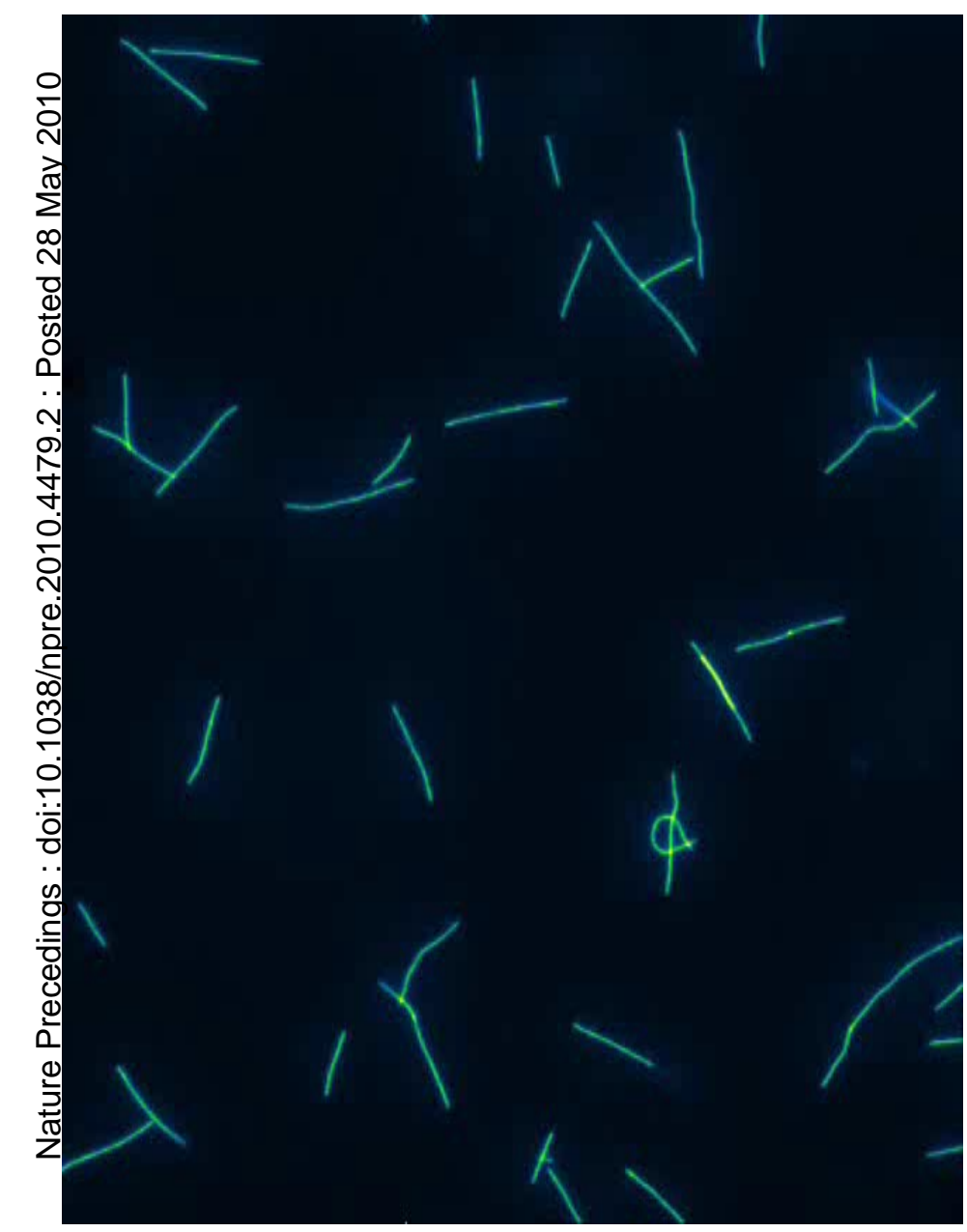

Also, noticed other features (preliminarily):

Reduced photobleaching

Longer-lasting MTs

MT-MT sticking

It turns out there is existing literature to support all of these observations 


\section{Heavy-hydrogen water has significantly different properties from "regular" water}

Naturally abundant 1 / 6600 hydrogen molecules is deuterium

17 mM deuterium in "standard mean ocean water"

$11 \%$ denser than $\mathrm{H}_{2} \mathrm{O}$. Freezes at 3.8C. D-bonds stronger.

Toxic to eukaryotes. The toxic effects are similar to chemotherapeutic drugs.

$\mathrm{D}_{2} \mathrm{O}$ has been used to stabilize viral vaccines.

$\mathrm{D}_{2} \mathrm{O}$ stabilizes tubulin and microtubules.

$\mathrm{D}_{2} \mathrm{O}$ stimulates tubulin assembly formation.

(Other fascinating factoids...)

Effects on kinesin motility has not yet been studied

Interesting ... but very complicated 


\section{Surprisingly, heavy-oxygen water has similar effect on gliding speed}

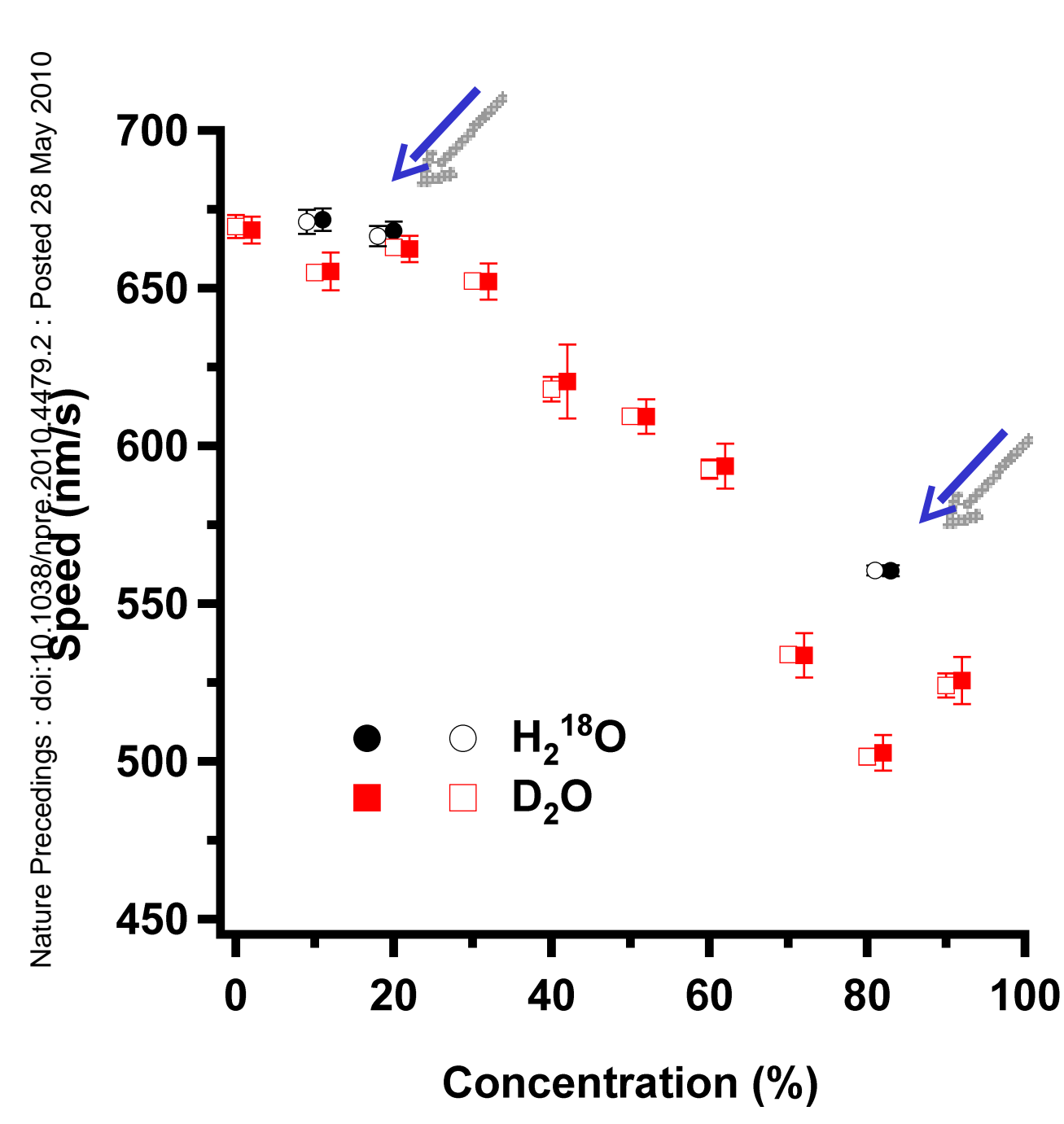

Chemistry of oxygen-18 water similar to regular water

Viscosity of oxygen-18 water only $5 \%$ higher than regular water (23\% for D2O)*

Heavy-O water much less confusing, possibly more tractable theoretically. Expensive, though $(\$ 1,000 /$ gram $)$

Possible that Heavy-O water is a good probe of kinesin-MT surface interaction

"Viscosity: Kudish et al. J. Chem. Soc., Faraday Trans. 1, 1972, 68, 2041 - 2046, DOI: $10.1039 / \mathrm{F} 19726802041$ 
Betaine (osmolyte) substantially reduces gliding speed

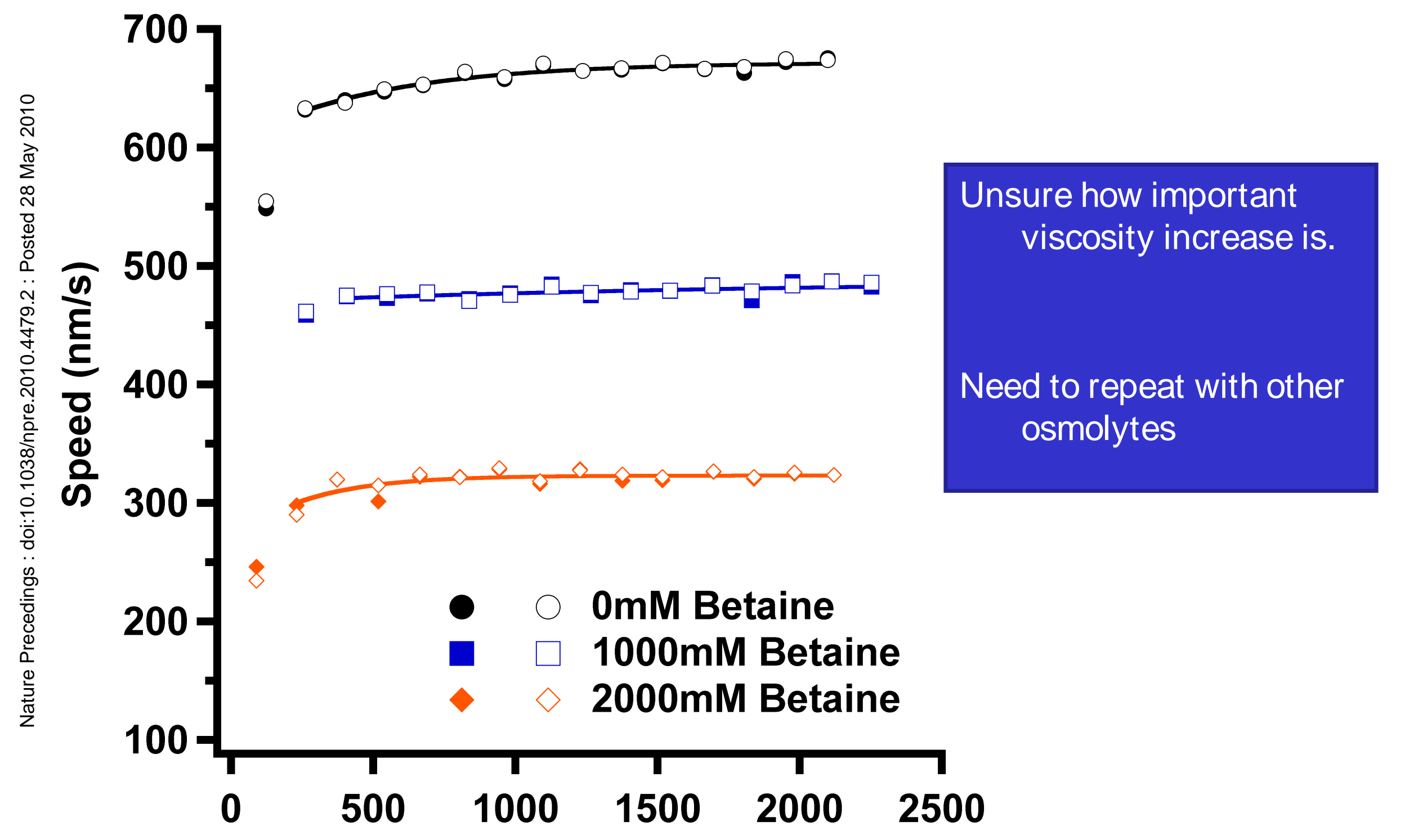

Time (s) 


\section{Current conclusions from water and other experiments}

Heavy-oxygen water looks like promising probe of hydration effects

Heavy-hydrogen water may also be interesting probe, but more complicated. May have benefits for stabilization of protein in the lab or in microdevices.

Betaine has strong effect on gliding speed. Viscosity a confounding variable. Need to compare with other osmolytes.

Need a better grasp of solution thermodynamics and kinetics and effect on motor proteins.

Type of surface passivation affects gliding speed. Gliding speed also increases with time. 


\section{Open Data, Open-source Software}

All data presented here is CCO-licensed and available online:

http://openwetware.org/wiki/Koch_Lab:Data/MT_Gliding_Assay_Readme_File
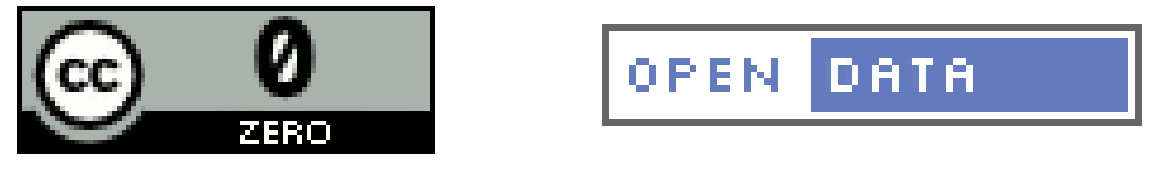

We're still working on the organization and how to best share. In the meantime:

If useful to you, get in touch and we can help you with the software and / or figuring out the data sets! 


\section{Acknowledgments}

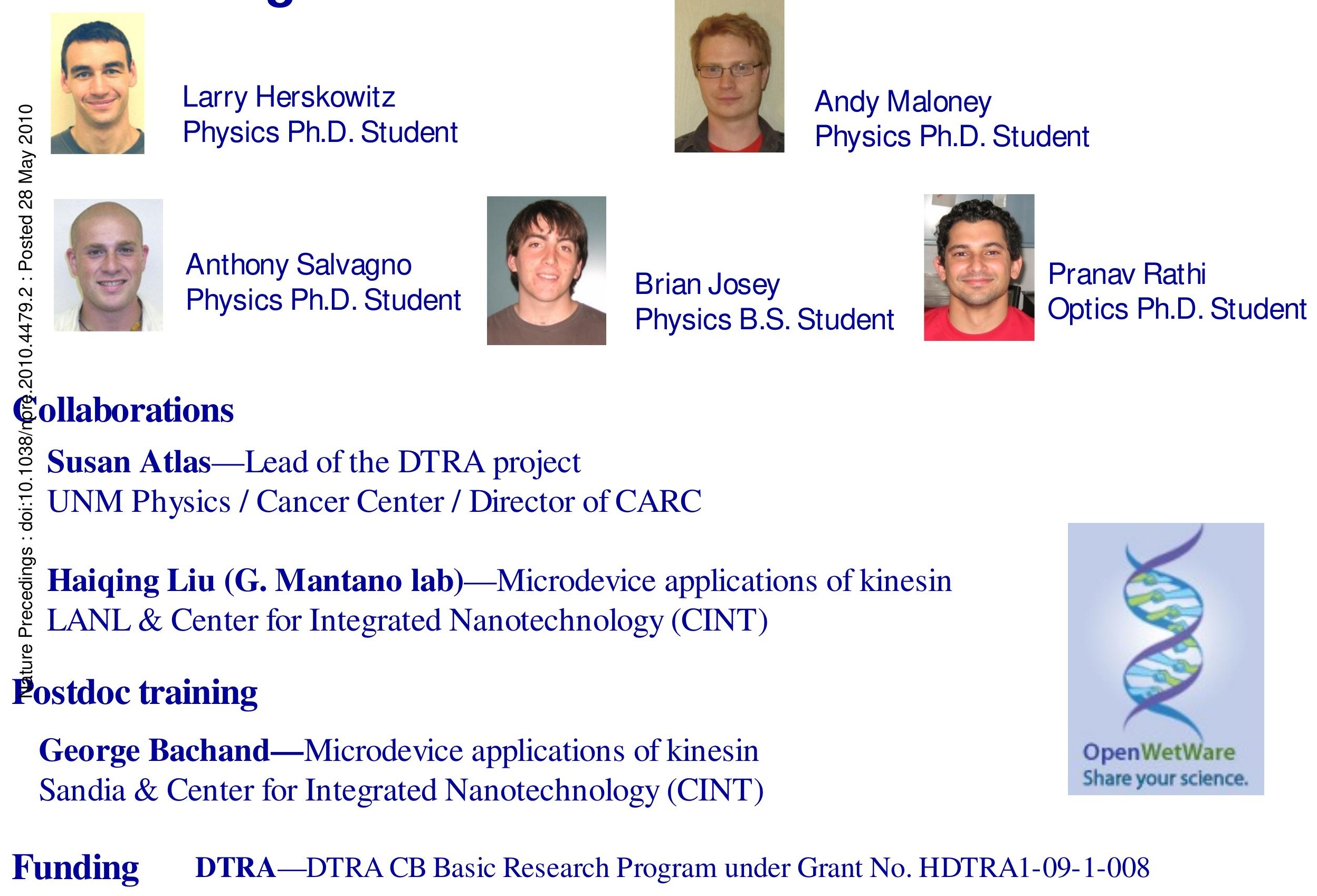

